\title{
Analysis of liquid sodium purity by Laser-Induced Breakdown Spectroscopy. Modeling and correction of signal fluctuation prior to quantitation of trace elements.
}

\author{
Cécile Maury ${ }^{\mathrm{a}, *}$, Jean-Baptiste Sirven ${ }^{\mathrm{a}, * *}$, Michel Tabarant ${ }^{\mathrm{a}}$, Daniel L'Hermite ${ }^{\mathrm{a}}$, \\ Jean-Louis Courouau ${ }^{\mathrm{a}}$, Catherine Gallou ${ }^{\mathrm{a}}$, Nadège Caron ${ }^{\mathrm{a}}$, Gilles Moutiers ${ }^{\mathrm{a}}$, Valérie \\ Cabuil $^{\mathrm{b}}$ \\ ${ }^{a}$ CEA, DEN, Department of Physical Chemistry, F-91191 Gif-sur-Yvette, France \\ ${ }^{b}$ UPMC Univ Paris 06, UMR 7195 PECSA, Physico-chemistry of Electrolytes, Colloids, Analytical \\ Sciences, F-75005 Paris, France
}

\begin{abstract}
Liquid sodium is used as coolant in sodium-cooled fast nuclear reactors. Among many parameters to monitor to ensure the safe operation of the reactor, the coolant chemical purity is a relevant indicator of several undesirable situations, like corrosion of structural materials or sodium contamination, which may release different elements in the coolant. Several techniques have already been implemented to measure the sodium purity, but their response time is long and not suited for continuous monitoring. Therefore, as a complement to them, Laser-Induced Breakdown Spectroscopy (LIBS) is considered as a promising technique for real-time analysis of the coolant purity. In this paper we report on the first LIBS quantitative measurements performed in liquid sodium at $150^{\circ} \mathrm{C}$. Calibration curves were traced for lead and indium using the standard addition method. Important intensity drifts and fluctuations were observed, mostly due to pressure variations in the sodium oven. Background subtraction and/or normalization was used to compensate for those intensity fluctuations. To describe the effect of these corrections on the analytical signal noise, a simple model was proposed and its results were found to satisfactorily fit the experimental data. Using this approach, the best detection limits were obtained for the background-subtracted and normalized data, and were found to be $6 \mathrm{ppm}$ for lead and $5 \mathrm{ppm}$ for indium.
\end{abstract}

Keywords: laser-induced breakdown spectroscopy, LIBS, liquid sodium, limit of detection, drift, noise, signal correction

\section{Introduction}

The Sodium Fast nuclear Reactors (SFR) are being evaluated in France as a generation IV system, as they benefit from the largest technological experience and are

\footnotetext{
*Principal corresponding author

** Corresponding author

Email addresses: cecile.maury@cea.fr (Cécile Maury), jean-baptiste.sirven@cea.fr (Jean-Baptiste Sirven)

Preprint submitted to Elsevier

December 6, 2012
} 
considered as one of the most promising systems. The roadmap is set in for the design, construction and operation of a prototype reactor, called ASTRID, in the 2020 decade.

Among the large number of parameters to monitor to ensure the safe operation of the reactor, the coolant chemical purity is a relevant indicator, primarily to maintain the coolant fluidity for heat-transfer purposes and to avoid pipe plugging, but also to detect phenomena like corrosion of structural materials by oxygen, contamination of the liquid metal (e.g. due to an air ingress), or even fuel clad failures. Each of these events may release in the sodium different elements such as oxygen, corrosion products ( $\mathrm{Fe}, \mathrm{Cr}, \mathrm{Ni}$ or $\mathrm{Mn}$ ) or fission products [1]. Monitoring the concentration of these elements in the coolant is then a way to limit to a minimum and to detect those undesirable situations.

In the French SFRs Phénix and SuperPhénix, the sodium purity was measured using plugging indicators and sodium samples (Tastena concept). Plugging indicators are based on the variation of impurities solubility with temperature [2]. The measurement is robust but not chemically selective, slow when the sodium purity is high, and it was found complex to interpret by the operator. Tastena measurements consist in sampling a few grams of liquid sodium, dissolving the sample and making a complete chemical analysis using standard laboratory techniques (e.g. ICP). The main limitation of this second measurement is that it is performed only a few times per cycle, most often during a reactor shut-down.

These techniques have been proved to be rather efficient for the operation of built reactors, and a large operating feedback was acquired on them. Yet the monitoring of the liquid sodium chemistry remains challenging. An ideal instrumentation should be implementable on-line, able to operate in severe conditions of high temperature and chemically reductive liquid sodium, and specific to chemical species present at the trace level. This may be useful to the reactor's operation in order to faster diagnostics of any chemical deviations and to propose corrective actions.

Therefore, as a complement to plugging indicators and Tastena sampling, there is an opportunity for innovative techniques that would give lower limits of detection, faster analysis, provide redundancy for safety related instrumentation, and be less complex compared to existing systems. In particular, real time measurements may have a strong interest to continuously monitor the coolant purity and to detect potential drifts of the target elements concentration. The laser-induced breakdown spectroscopy (LIBS) technique is developed at CEA for that purpose. In LIBS, a pulsed laser is focalized onto the sample surface. The resulting laser ablation leads to the formation of a plasma. The spectroscopic analysis of the plasma emission allows to measure the sample elemental composition.

As an all-optical technique, LIBS is well suited to remote or on-line analysis in hostile conditions [3] [4]. For that reason, it has found several applications in nuclear environment, where radioactivity [5], high temperature [6], presence of highly corrosive media [7] or weak accessibility to the sample [8] strongly limit the choice of analytical techniques.

In this paper, LIBS was used to analyze the chemical purity of liquid sodium, with the objective to detect in real-time a possible drift of the metallic impurities concentration in the primary cooling circuit. As a first step, our work consisted in characterizing this approach and in estimating the measurement sensitivity for two model elements highly soluble in liquid sodium: lead and indium [9]. Acquisition parameters were optimized to lower as much as possible their detection limits, and calibration curves were obtained using the standard addition method. Strong signal drifts and variations were observed. 
Different approaches were tested to model them and to normalize the analytical signal in order to improve the calibration.

\section{Experimental setup and data acquisition}

\subsection{Description of the experimental setup}

The sample consisted of $300 \mathrm{~g}$ of pure metallic sodium, contained in a hermetic oven topped with 3 optical windows. A heating system was used to heat the sodium at $150^{\circ} \mathrm{C}$, i.e. $52^{\circ} \mathrm{C}$ above its melting point. The sodium sample was under an ultra-pure argon atmosphere representative of a sodium-cooled reactor. In order to protect the optical windows from possible projections of ablated matter and condensation of sodium vapor, argon was flushed below each of them with a controlled flow rate of $7 \mathrm{~L} / \mathrm{min}$.

We chose lead $(\mathrm{Pb})$ and indium $(\mathrm{In})$ as analytes since they are highly soluble in liquid sodium at $150^{\circ} \mathrm{C}[9]$ and have emission lines in the UV-visible range [10]. Furthermore, their concentration was varied in the sample using the standard addition method [11]. Solid pieces of pure lead and indium were weighed and successively introduced in the oven. We assumed that both metals were totally dissolved and that the obtained mixture was homogeneous. These hypotheses were verified: after the experiments several samples were taken from the mixture and analysed using ICP-AES, which showed the validity of our assumptions.

The experimental setup is shown on Figure 1. A quadrupled Nd:YAG laser (266 $\mathrm{nm}, 5 \mathrm{~ns}$ pulse width, $20 \mathrm{~Hz}$ repetition rate) was used to ablate the sodium sample. It was chosen to perform LIBS ablation because liquid sodium absorbs more efficiently UV radiations. It could operate at $15 \mathrm{~mJ}$ maximum energy and was followed by an energy attenuator. The ablation beam was focused with normal incidence onto the liquid sodium surface using a convergent lens (focal length $1 \mathrm{~m}$ ). The laser spot on liquid sodium had a diameter of $500 \mu \mathrm{m}$. The working irradiance was $1.3 \mathrm{GW} / \mathrm{cm}^{2}$.

The LIBS signal was collected in the same direction as the ablation beam, using a telescope (working distance $950 \mathrm{~mm}$ ), and sent to the detection system using an optical fiber (numerical apperture of 0.22 and core diameter $910 \mu \mathrm{m}$ ). A Czerny-Turner monochromator (Acton SP2300i, 3600 grooves $/ \mathrm{mm}$ ) was used for the analysis of the LIBS signal. The entrance slit was $100 \mu \mathrm{m}$ wide. The spectra were recorded using an

Andor iStar ICCD $(512 \times 2048$ pixels $)$. This system had a resolution power $\frac{\lambda}{\Delta \lambda}$ of 3000 at $283 \mathrm{~nm}$.

Four hundred laser shots were accumulated for each spectrum. For each standard addition, 100 replica were successively acquired in about $30 \mathrm{~min}$. Figure 2 shows typical spectra, obtained using optimal acquisition parameters (see paragraph 2.2) for a concentration of $89 \mathrm{ppm}$ of lead and $50 \mathrm{ppm}$ of indium in liquid sodium. Atomic lines are visible above a continuum which can be attributed to Bremsstrahlung, radiative recombinations and in the case of $\mathrm{Pb}$, the wing of a strong sodium line at $285.3 \mathrm{~nm}$.

\subsection{Optimization of acquisition parameters}

The limit of detection ( $\mathrm{LoD}$ ) is linked to the continuum background noise, then to the signal to background noise ratio (SNR) [12]. To decrease the LoD is equivalent to increase the SNR. Therefore, prior to the calibration, a certain mass of lead and 


\begin{tabular}{|c|ccc|ccc|}
\hline Analytes & \multicolumn{3}{|c|}{$\mathrm{Pb}$} & \multicolumn{3}{|c|}{ In } \\
\hline$\lambda(\mathrm{nm})$ & 405.8 & 368.3 & 283.3 & 451.1 & 410.2 & 325.6 \\
\hline$S$ & $2.710^{7}$ & $2.110^{7}$ & $2.310^{7}$ & $3.710^{7}$ & $2.610^{7}$ & $1.410^{7}$ \\
$B_{1}+B_{2}$ & $3.610^{7}$ & $2.410^{7}$ & $2.510^{7}$ & $1.010^{8}$ & $7.410^{7}$ & $2.410^{7}$ \\
$\sigma\left(B_{1}+B_{2}\right)$ & $1.410^{6}$ & $1.610^{6}$ & $6.410^{5}$ & $3.810^{6}$ & $3.610^{6}$ & $1.510^{6}$ \\
\hline$S N R$ & 20 & 13 & 37 & 9.7 & 7.3 & 9.2 \\
\hline
\end{tabular}

Table 1: Values of the background-subtracted intensity $S$ (net signal), the total background intensity $B_{1}+B_{2}$ (see paragraph 3.1), the background noise $\sigma\left(B_{1}+B_{2}\right)$ and the signal to noise ratio $S N R=$ $S / \sigma\left(B_{1}+B_{2}\right)$ for several $\mathrm{Pb}$ and In lines. The data were obtained from a sodium sample with $1365 \mathrm{ppm}$ of $\mathrm{Pb}$ and $50 \mathrm{ppm}$ of In. The spectra were acquired with a $1 \mu$ s delay and a $1 \mu$ s gate width in the $\mathrm{Pb}$ case, and a $4 \mu$ s delay and a $6 \mu$ s gate width in the case of In.

indium was introduced in liquid sodium, sufficient to give a signal well above the limit of detection. The corresponding concentrations were respectively $1365 \mathrm{ppm}$ and $50 \mathrm{ppm}$. Then acquisition parameters were optimized so that the SNR is maximum. As the lead concentration was rather high compared to the calibration range (see section 3.2), we checked that self-absorption could be neglected at this concentration. Therefore the results of this optimization were valid even at concentrations lower than $100 \mathrm{ppm}$.

We first chose the emission lines giving the highest SNR for both analytes. Table 1 sums up the signal, $S$, the background intensity, $B_{1}+B_{2}$ (see paragraph 3.1 for notations), the background noise $\sigma\left(B_{1}+B_{2}\right)$ and the SNR obtained for different atomic lines of lead and indium. For both elements the background noise was found to increase with wavelength. The lead line giving the highest SNR was the $283.3 \mathrm{~nm}$ line. Therefore, it was selected to draw the lead calibration curves (see paragraph 3.2). For indium the SNR was not very different for the three measured lines. Yet we naturally chose to work with the one giving the highest SNR. Therefore, the $451.1 \mathrm{~nm}$ line was selected to draw the indium calibration curve (see paragraph 3.2).

Secondly, we studied the evolution of the SNR as a function of the ablation pulse energy for the $283.3 \mathrm{~nm}$ line of lead. The highest SNR was obtained for the maximum available pulse energy $(15 \mathrm{~mJ})$.

The last step consisted in optimizing the acquisition time gate for both elements. For that purpose, we studied the evolution of the SNR as a function of the acquisition delay. The gate width was set to $1 \mu s$ and the gate delay was varied by $1 \mu s$ steps. We can see on Figure 3 that for both elements, the SNR increases during the first few microseconds. It then attains a plateau before dropping. In the $\mathrm{Pb}$ case, the point corresponding to the $2 \mu s$ delay is abnormally high for unknown reasons. From these data, the optimal gate delay and width were fixed respectively at $4 \mu s$ and $6 \mu s$ for both elements to obtain the highest SNR.

\subsection{Data acquisition}

Spectra were acquired using the standard addition method and the optimal acquisition parameters determined previously were used to plot calibration curves. However, they showed strong variations of the global intensity, as can be seen on Figure 4, as well as intensity drifts during the acquisition a series of 100 spectra as can be seen on Figure 5 . 
To explain this observation several tests were done on the detection setup repeatability, on operating conditions and on the dissolution kinetics of standard additions. None of these factors was found to account for these fluctuations. We finally attributed them to pressure variations in the oven. Indeed, in our setup, only the argon in-flow rate could be controlled, not its pressure in the oven. During the acquisitions, we observed the slow formation of sodium corks in the outlet circuit. They were the result of the condensation of sodium vapour dragged into the circuit by the argon flow. Pressure variations could change the plasma confinement, therefore its temperature and emission intensity [13]. To ensure this, we varied the pressure inside the oven using an adjustable valve at the oven outlet and a pressure gauge. To avoid possible sodium corks, LIBS measurements were performed in a short time (a few minutes) between 1 and 1.4 bar, which seemed to us a maximum reasonable pressure that we might have reached during the experiments. As shown on Figure 6, in this pressure range the line and the background intensity significantly decrease when the pressure increases. This variation is consistent with that observed on Figure 4, which validates our hypothesis. The signal to background ratio remains relatively constant over the same range. This introduces the signal normalization by the background intensity, developed in section 3 , in order to compensate for the observed fluctuations.

During the series of 100 spectra recorded for each standard addition, we also identified fast intensity variations from one spectrum to another. Like fluctuations from one standard addition to another, those were proportional ones, i.e. global intensity variations. The shape of spectra did not vary, only their amplitude did. This fast noise, referred to as source noise, is due to laser energy variations, as well as fluctuations of laser-plasma and laser-ablated particles interactions (see figure 5).

Finally, in the case of lead, as can be seen on Figures 2 and 4, the $283.3 \mathrm{~nm}$ line lies on the wing of a strong self-absorbed sodium line at $285.3 \mathrm{~nm}$. We found that the contribution of the sodium line to the lead line background fluctuation is uncorrelated with the drifts and intensity variations described above. This means that the sodium $285.3 \mathrm{~nm}$ line undergoes an additional noise which is due neither to pressure fluctuations nor to the source noise. As sodium is the most abundant element in the plasma, this additional variation might be attributed to non linear fluctuations due to self-absorption [14].

\section{Results and discussion}

\subsection{Correction of the analytical signal noise}

The drifts, intensity variations, source noise and background fluctuations, can be compensated for by processing the spectral data in different ways. A widespread one in optical emission spectroscopy consists in using a matrix element line to normalize the spectra. However, in our case, the sodium lines were strongly self-absorbed and could not be used for that purpose. Instead, we considered several approaches based on the continuous background. Then we studied the following corrections of the analytical line signal: background subtraction, background normalization, and the combination of these two corrections. These operations aimed at decreasing the analytical signal noise, hence improving the detection limit. Therefore, we built a simple model of the signal noise, which was then used to determine the best way to correct intensity drifts and global intensity variations. 
The line intensity $I$ is expressed as $I=S+B_{1}+B_{2}$, where $S$ is the backgroundsubtracted line intensity, $B_{1}$ is the continuum intensity at the line wavelength, and $B_{2}$ is the sodium $285.3 \mathrm{~nm}$ line wing contribution to the line background (see figure 2). In the case of indium, $B_{2}=0$. We also define the background intensity $B_{3}$ to normalize the signal. $B_{3}$ is calculated at a wavelength distant from any sodium line.

Each of the above described quantities was subjected to several noise sources:

- Global intensity variations, due to drifts and to the source noise, induce proportional intensity fluctuations both for lines and backgrounds. Those noises are labelled $\sigma_{G}$, and we define proportionality relations between them such that $\sigma_{G}(I)=\alpha \sigma_{G}\left(B_{3}\right), \sigma_{G}\left(B_{1}\right)=\beta \sigma_{G}\left(B_{3}\right), \sigma_{G}\left(B_{2}\right)=\gamma \sigma_{G}\left(B_{3}\right)$ and $\sigma_{G}(S)=\delta \sigma_{G}\left(B_{3}\right)$, where $\alpha, \beta, \gamma$ and $\delta$ are constant and $\alpha=\beta+\gamma+\delta$, where $\alpha$ and $\delta$ depend on the analyte concentration in the sample.

- The shot noise $\sigma_{N}$ which is Poissonnian.

- The matrix element line wing fluctuations, which only affect $B_{2}$ in the case of lead, and generate a noise labelled $\sigma_{M}$ such that $\sigma_{M}(I)=\sigma_{M}\left(B_{2}\right)$ and $\sigma_{M}\left(B_{1}\right)=$ $\sigma_{M}\left(B_{3}\right)=0 . \sigma_{M}\left(B_{2}\right)$ is supposed to be uncorrelated to $\sigma_{G}\left(B_{2}\right)$, i.e. it is not a proportional fluctuation.

Then the total noise can be expressed as $\sigma(i)=\sqrt{\sigma_{G}(i)^{2}+\sigma_{N}(i)^{2}+\sigma_{M}(i)^{2}}$. From this, we obtain (see Appendix for details):

$$
\begin{gathered}
\sigma(S)^{2}=\sigma(I)^{2}-\sigma_{M}(I)^{2}+\sigma_{N}\left(B_{1}+B_{2}\right)^{2}-\left(\alpha^{2}-\delta^{2}\right) \sigma_{G}\left(B_{3}\right)^{2} \\
\sigma\left(I / B_{3}\right)^{2}=\frac{1}{B_{3}{ }^{2}}\left[\sigma(I)^{2}+\alpha^{2} \sigma_{N}\left(B_{3}\right)^{2}-\sigma_{G}(I)^{2}\right] \\
\sigma\left(S / B_{3}\right)^{2}=\frac{1}{{B_{3}}^{2}}\left[\sigma(I)^{2}+\sigma_{N}\left(B_{1}+B_{2}\right)^{2}+\delta^{2} \sigma_{N}\left(B_{3}\right)^{2}-\sigma_{M}(I)^{2}-\sigma_{G}(I)^{2}\right]
\end{gathered}
$$

Where $\sigma_{N}\left(B_{1}+B_{2}\right)^{2}=\sigma_{N}\left(B_{1}\right)^{2}+\sigma_{N}\left(B_{2}\right)^{2}$. From Equation 1, considering the net signal noise $\sigma(S)$, we see that the background subtraction of the raw intensity removes the background fluctuations due to the sodium line wing $\sigma_{M}(I)$. However it adds the background shot noise and does not entirely correct the global intensity variations. From Equation 2, looking at the $\sigma\left(I / B_{3}\right)$ expression, we see that normalizing the raw line intensity corrects the global intensity variations $\sigma_{G}(I)$, but not the background intensity fluctuations due to the sodium line wing $\left(\sigma_{M}\right.$ term). It also adds some shot noise. Finally, as shown by Equation 3 for the expression of $\sigma\left(S / B_{3}\right)$, normalizing the backgroundsubtracted line intensity enables to eliminate global intensity variations and background intensity fluctuations due to the sodium line wing. However, each operation (background subtraction and normalization) adds shot noise related to both backgrounds, the line one, $B_{1}+B_{2}$, and the normalization one, $B_{3}$. From these equations, it is clear that background subtraction and normalization are interesting ways to reduce the analytical signal noise only when this noise is not dominated by shot noise.

Then, we applied our model to our experimental data. Figure 7 compares the model results and the experimental noises for different concentrations of lead and indium. For indium, the agreement between both approaches is good, despite a few outliers such as the $77 \mathrm{ppm}$ points for the last two cases. For lead, the model yields correct results for $\sigma(S)$ and $\sigma\left(I / B_{3}\right)$, but it significantly underestimates $\sigma\left(S / B_{3}\right)$. In other words, the 
experimental correction of intensity variations by the normalization and the background subtraction is not as good as what the model predicts. This could mean that for lead there is another, unidentified noise source, affecting the net line intensity signal or the background intensity, which is not taken into account by the model. However, we should then observe a discrepancy also on the $S$ or on the $I / B_{3}$ data, which is not the case. The fact that the lead line lies on the wing of a fluctuating sodium line might play a role, but again in this case we should observe a higher discrepancy on the results obtained for the normalized intensity. The reason of this underestimation remains unclear.

The main interest of this model is that from the raw experimental data, it allows to determining the best way to compensate for observed fluctuations. Therefore, it is useful to correct possible drifts, then to improve the analytical signal repeatability and trueness.

In the following paragraph, calibration curves of lead and indium are presented, using the different signal correction methods studied in this paragraph.

\subsection{Calibration curves}

In order to calculate the net signal $S$, the normalized raw intensity $I / B_{3}$ and the normalized net signal $S / B_{3}$, the raw line intensity $I$ was first computed as the sum over the full width at half maximum (21 pixels). Then, we estimated the line background $B$ using a linear fit for indium or a quadratic fit for lead, calculated from spectral zones on both sides of the line. The choice of the background estimation procedure was driven by the spectrum profile in the vicinity of the emission line (see figure 2). However, in every case, the background intensity was integrated over 21 pixels. The normalization background $B_{3}$ was calculated on the edge of the spectra, around $273 \mathrm{~nm}$ in the case of lead, and around $453 \mathrm{~nm}$ in the case of indium.

The calibration lines obtained are shown on figure 8. For each standard addition the 100 spectra were accumulated ten-by-ten to obtain 10 replicas of 4000 laser shots. Prediction bands were drawn for a 95\% confidence level using those 10 replicas per concentration [15] [16].

As can be seen on figure 8, for the $S$ calibration curve, the $77 \mathrm{ppm}$ point of the indium curve and the $33 \mathrm{ppm}$ point of the lead curve are clearly out of the prediction bands, which means that the background subtraction is not sufficient to compensate for signal fluctuations. This is consistent with the fact that the net line intensity $S$ undergoes variations due to pressure fluctuations, as shown on Figure 5, and that the background subtraction does not completely correct those variations (as predicted by equations 3 ).

The linearity of the $I / B_{3}$ calibration curve is improved compared to that of the $S$ one and prediction bands are closer together. This is particularly true for the indium curve, for which the $77 \mathrm{ppm}$ point is perfectly corrected by the normalization. The linearity of the $S / B_{3}$ calibration curve is still improved compared to that of both $S$ and $I / B_{3}$. The prediction bands are still closer together. There is no outlier on the lead curve, and only one on the indium one. The calibration curves obtained from the $S / B_{3}$ data are clearly the best. This result is in good agreement with the model.

The limits of detection (LoD) were calculated from the calibration curves using the prediction bands method, which takes into account the uncertainty on the calibration line slope and on its intercept point with the signal axis. In other words, not only the signal and background repeatability is used to estimate the LoD, but also the calibration 


\begin{tabular}{|c|c|c|}
\hline Signal correction & \multicolumn{2}{|c|}{ LoD (ppm) } \\
\cline { 2 - 3 } method & $\mathrm{Pb}$ & $\mathrm{In}$ \\
\hline$S$ & 13 & 25 \\
$I / B_{3}$ & 10 & 7 \\
$S / B_{3}$ & 6 & 5 \\
\hline
\end{tabular}

Table 2: Limits of detection of lead and indium obtained from the background-subtracted and/or normalized intensity data. See paragraph 3.2 for notations.

data linearity. The lead and indium LoDs obtained from the six calibration curves are compiled in table 2. Clearly, the lowest LoDs were obtained from the $S / B_{3}$ data, which is also consistent with the model.

The significant decrease of the indium LoD from the $S$ to $I / B_{3}$ data is due to the compensation of the $77 \mathrm{ppm}$ point by the normalization, which leads to closer prediction bands. The improvement for the $I / B_{3}$ compared to the $S / B_{3}$ data is less significant. Indeed, in the case of indium, there are no matrix line fluctuations and the normalization of the raw intensity and that of the background-subtracted signal yield similar results. In the case of lead, we note a steady decrease of the LoD from the $S$ to the $S / B_{3}$ data, which is in agreement with the model.

\section{Conclusions}

In Sodium-cooled Fast nuclear Reactors, the sodium chemical purity has to be periodically measured to monitor the reactor operation in terms of corrosion, leakages between cooling circuits, or even fuel clad failures. Laser-induced breakdown spectroscopy is considered as an innovative online technique for this application, complementary to already established methods.

In this work, we performed the analysis of liquid sodium purity by LIBS. Lead and indium were chosen as analytes since they are easily soluble in sodium. The standard addition method was used for calibration and LIBS measurements were performed at $150^{\circ} \mathrm{C}$ on the static liquid surface. Experimental parameters were optimized to maximize the signal to background noise ratio.

Important signal fluctuations were identified, mostly due to pressure variations in the sodium oven. Different approaches were considered to correct the line signal from these variations, and we proposed a simple model to describe their effect on the analytical signal noise. This model was found to correctly fit the experimental data for indium. For lead, results were less good, presumably due to the presence of a sodium line wing interfering with the lead line and inducing additional fluctuations that were not taken into account in the model. More generally, this model can be applied when intensity drifts are observed and cannot be experimentally compensated for (e.g. using a pressure controller), in order to determine the best way to correct the signal and to improve the detection limit.

As predicted by the model, the best calibration curves were obtained using background subtraction and background normalization of the line intensity. In this case, the detection limits were respectively $6 \mathrm{ppm}$ and $5 \mathrm{ppm}$ for lead and indium. 
Finally, this work fully validated our analytical approach on two model elements. Now our objective is to measure elements more representative of the investigated phenomena in liquid sodium, like corrosion. Yet these elements have a much lower solubility limit. Therefore, the measurement sensitivity should be significantly improved. The laser ablation - laser-induced fluorescence technique (LA-LIF) is currently studied in our laboratory to achieve this goal.

\section{Appendix}

This appendix presents the detailed calculations performed to obtain equations 1,2 and 3 .

The raw line intensity is expressed as $I=S+B_{1}+B_{2}$, where $B_{1}$ and $B_{2}$ are defined in paragraph 3.1. A few hypotheses are made, mainly the proportionality relations between the mean values: $I=\alpha B_{3}, B_{1}=\beta B_{3}, B_{2}=\gamma B_{3}$ and $S=\delta B_{3}$, which lead to proportionality relations between the noises: $\sigma_{G}(I)=\alpha \sigma_{G}\left(B_{3}\right), \sigma_{G}\left(B_{1}\right)=\beta \sigma_{G}\left(B_{3}\right)$, $\sigma_{G}\left(B_{2}\right)=\gamma \sigma_{G}\left(B_{3}\right)$ and $\sigma_{G}(S)=\delta \sigma_{G}\left(B_{3}\right)$, and allow the expression of all the quantities as:

$$
\left\{\begin{aligned}
I & =\alpha B_{3}+\varepsilon_{I}+\eta \\
B_{1} & =\beta B_{3}+\varepsilon_{1}+\eta \\
B_{2} & =\gamma B_{3}+\varepsilon_{2}+\eta
\end{aligned}\right.
$$

were $\varepsilon_{i}$ and $\eta$ are random fluctuations whose mean are equal to $0 . \varepsilon_{i}$ is the fluctuation due to the shot noise corresponding to the quantity i, while $\eta$ is the fluctuation due to the matrix element line. They follow the relationships:

$$
\left\{\begin{array}{l}
\operatorname{cov}\left(\varepsilon_{i}, \varepsilon_{i}\right)=\sigma_{N}(i)^{2} \\
\operatorname{cov}\left(\varepsilon_{i}, \varepsilon_{j}\right)=0 \\
\operatorname{cov}(\eta, \eta)=\sigma_{M}(I)^{2} \\
\operatorname{cov}\left(\varepsilon_{i}, \eta\right)=0 \\
\operatorname{cov}\left(B_{3}, B_{3}\right)=\sigma_{G}\left(B_{3}\right)^{2}
\end{array}\right.
$$

In addition, for any quantities $\mathrm{A}$ and $\mathrm{B}$ we can write [17].

$$
\begin{gathered}
\sigma(A-B)^{2}=\sigma(A)^{2}+\sigma(B)^{2}-2 \operatorname{cov}(A, B) \\
R S D\left(\frac{A}{B}\right)=\left[\frac{\sigma(A)^{2}}{A^{2}}+\frac{\sigma(B)^{2}}{B^{2}}-\frac{2}{A B} \operatorname{cov}(A, B)\right]^{1 / 2}
\end{gathered}
$$

were $R S D(A)=\sigma_{A} /\langle A\rangle$.

\subsection{Background subtracted intensity}

The background-subtracted signal is $S=I-B_{1}-B_{2}$. Using equation 6 , its variations can be expressed as:

$$
\sigma(S)^{2}=\sigma(I)^{2}+\sigma\left(B_{1}\right)^{2}+\sigma\left(B_{2}\right)^{2}-2\left(\operatorname{cov}\left(I, B_{1}\right)+\operatorname{cov}\left(I, B_{2}\right)-\operatorname{cov}\left(B_{1}, B_{2}\right)\right)
$$


From equations 9 and 5, the covariances can be expressed as:

$$
\left\{\begin{array}{l}
\operatorname{cov}\left(I, B_{1}\right)=\alpha \beta \sigma_{G}\left(B_{3}\right)^{2} \\
\operatorname{cov}\left(I, B_{2}\right)=\alpha \gamma \sigma_{G}\left(B_{3}\right)^{2}+\sigma_{M}(I)^{2} \\
\operatorname{cov}\left(B_{1}, B_{2}\right)=\beta \gamma \sigma_{G}\left(B_{3}\right)^{2}
\end{array}\right.
$$

Using the expression of $\sigma(I), \sigma\left(B_{1}\right)$ and $\sigma\left(B_{2}\right)$, equation 8 can be reduced to:

$$
\sigma(S)^{2}=\sigma(I)^{2}-\sigma_{M}(I)^{2}+\sigma_{N}\left(B_{1}+B_{2}\right)^{2}-\left(\alpha^{2}-\delta^{2}\right) \sigma_{G}\left(B_{3}\right)^{2}
$$

\subsection{Background normalized intensity}

The background normalized signal is $I / B_{3}$. Its variations can be expressed, using equations 7 and 5 , as:

$$
\sigma\left(I / B_{3}\right)^{2}=\frac{1}{B_{3}{ }^{2}}\left[\sigma(I)^{2}+\alpha^{2} \sigma\left(B_{3}\right)^{2}-2 \alpha^{2} \sigma_{G}(I)^{2}\right]
$$

Using the expression of $\sigma(I), \sigma\left(B_{1}\right)$ and $\sigma\left(B_{2}\right)$, equation 11 can be rewritten as:

$$
\sigma\left(I / B_{3}\right)^{2}=\frac{1}{B_{3}{ }^{2}}\left[\sigma(I)^{2}+\alpha^{2} \sigma_{N}\left(B_{3}\right)^{2}-\sigma_{G}(I)^{2}\right]
$$

\subsection{Normalized background-subtracted intensity}

The normalized background-subtracted signal is $S / B_{3}$. Using equations 7 and 5 , its variations can be expressed as:

$$
\sigma\left(S / B_{3}\right)^{2}=\frac{1}{B_{3}{ }^{2}}\left[\sigma(S)^{2}+\delta^{2} \sigma\left(B_{3}\right)^{3}-2 \delta^{2} \sigma_{G}\left(B_{3}\right)^{2}\right]
$$

Using the expression of $\sigma(I), \sigma\left(B_{1}\right)$ and $\sigma\left(B_{2}\right)$, equation 13 can be rewritten as:

$$
\sigma\left(S / B_{3}\right)^{2}=\frac{1}{B_{3}{ }^{2}}\left[\sigma(I)^{2}+\sigma_{N}\left(B_{1}+B_{2}\right)^{2}+\delta^{2} \sigma_{N}\left(B_{3}\right)^{2}-\sigma_{M}(I)^{2}-\sigma_{G}(I)^{2}\right]
$$

\section{Bibliography}

\section{References}

[1] J-L. Courouau, Chapter 4: chemistry control and monitoring systems, in: Handbook on LeadBismuth Eutectic Alloy and Lead Properties, Materials Compatibility, Thermal-hydraulics and Technologies.. NEA/OECD. IAEA, Vienna, Autria, 2007.

[2] H. Yamamoto, M. Murase, I. Sumida, and K. Kotani. Measurement Of Impurity Concentration In Sodium By Automatic Plugging Indicator. Journal of Nuclear Science and Technology, 14 (1977) $452-456$.

[3] D.A. Cremers and L.J. Radziemski. Handbook of Laser Induced Breakdown Spectroscopy. John Wily \& Son LtD (England), 2006.

[4] A.W. Miziolek, V. Palleschi and I. Schechter. Laser Induced Breakdown Spectroscopy. Cambridge University Press, 2008.

[5] P. Fichet, P. Mauchien, and C. Moulin. Determination of impurities in uranium and plutonium dioxides by laser-induced breakdown spectroscopy. Appl. Spectrosc. 53 (1999) 1111-1117. 
[6] J.I. Yun, R. Klenze, and J.I. Kim. Laser-induced breakdown spectroscopy for the on-line multielement analysis of highly radioactive glass melt. Part 1: Characterization and evaluation of the method. Appl. Spectrosc. 56 (2002) 437-448.

[7] A. Sarkar, V.M. Telmore, D. Alamelu, and S.K. Aggarwal. Laser-induced breakdown spectroscopic quantification of platinium group metals in simulated high level nuclear waste. J. Anal. At. Spectrom. 24 (2009) 1545-1550.

[8] A.I. Whitehouse, J. Young, I.M. Botheroyd, S. Lawson, C.P. Evans and J. Wright. Remote material analysis of nuclear power station steam generator tubes by laser-induced breakdown spectroscopy. Spectrochim. Acta Part B 56 (2001) 821-830.

[9] H.U. Borgstedt and C.K. Mathews. Applied Chemistry of the Alkali Metals. Plenum Press, New York, 1987.

[10] NIST atomic spectra database, http://physics.nist.gov/physrefdata/handbook/periodictable.htm, 2008.

[11] L.H. Keith, W. Crummett, J. Deegan, R.A. Libby, J.K. Taylor, and . Wentler. Principles of environmental-analysis. Anal. Chem. 55 (1983) 2210-2218.

[12] L.A. Currie. Nomenclature in evaluation of analytical methods including detection and quantification capabilities (IUPAC Recommendations 1995). Anal. Chim. Acta 391 (1999) 105-126.

[13] E. Tognoni, V. Palleschi, M. Corsi, and G. Cristoforetti. Quantitative micro-analysis by laserinduced breakdown spectroscopy: a review of the experimental approaches. Spectrochim. Acta Part B 57 (2002) 1115-1130.

[14] J.A. Aguilera and C. Aragó n. Characterisation of laser-induced plasmas by optical emission spectroscopy: a review of experiments and methods. Spectrochim. Acta Part B 63 (2008) 893-916.

[15] A. Hubaux and G. Vos. Decision and detection limits for linear calibration curves. Anal.Chem. 42 (1970) 849-855.

[16] Jean-Michel Mermet. Limit of quantitation in atomic spectrometry: An unambiguous concept? Spectrochim. Acta Part B 63 (2008) 166-182.

[17] D.W. Hahn and N. Omenetto. Laser-Induced Breakdown Spectroscopy (LIBS), Part II: Review of Instrumental and Methodological Approaches to Matrial Analysis and Applications to Different Fields. Applied Spectroscopy 66 (2012) 347 - 419 


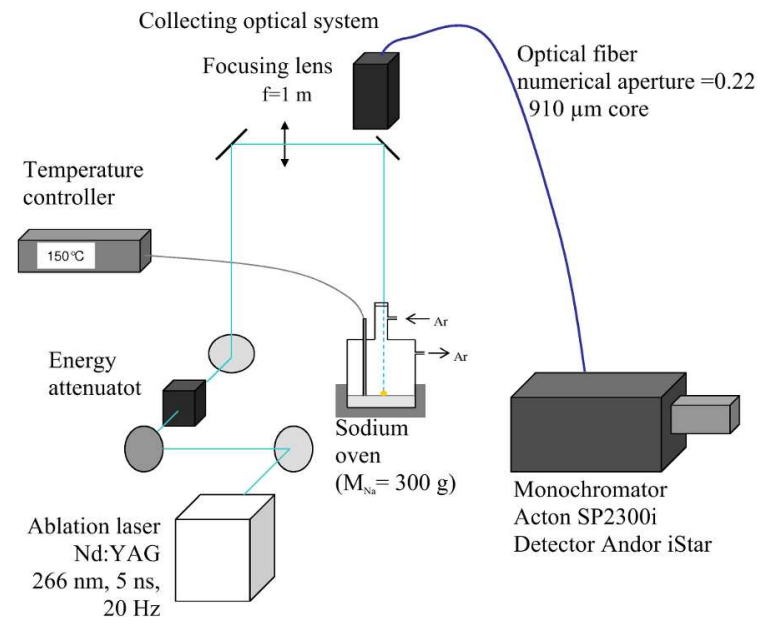

Figure 1: Experimental setup.
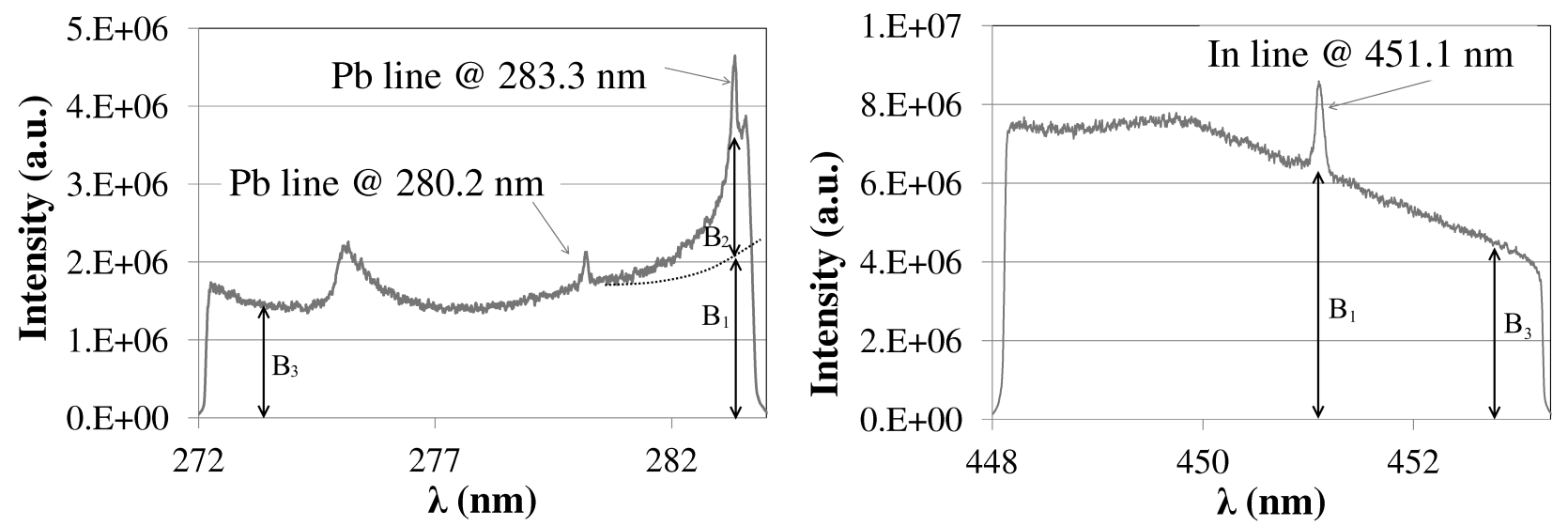

Figure 2: LIBS spectra acquired in the optimal conditions (see paragraph 2.2), obtained from a sample containing $89 \mathrm{ppm}$ of $\mathrm{Pb}$ and $50 \mathrm{ppm}$ of $\mathrm{In}$. 

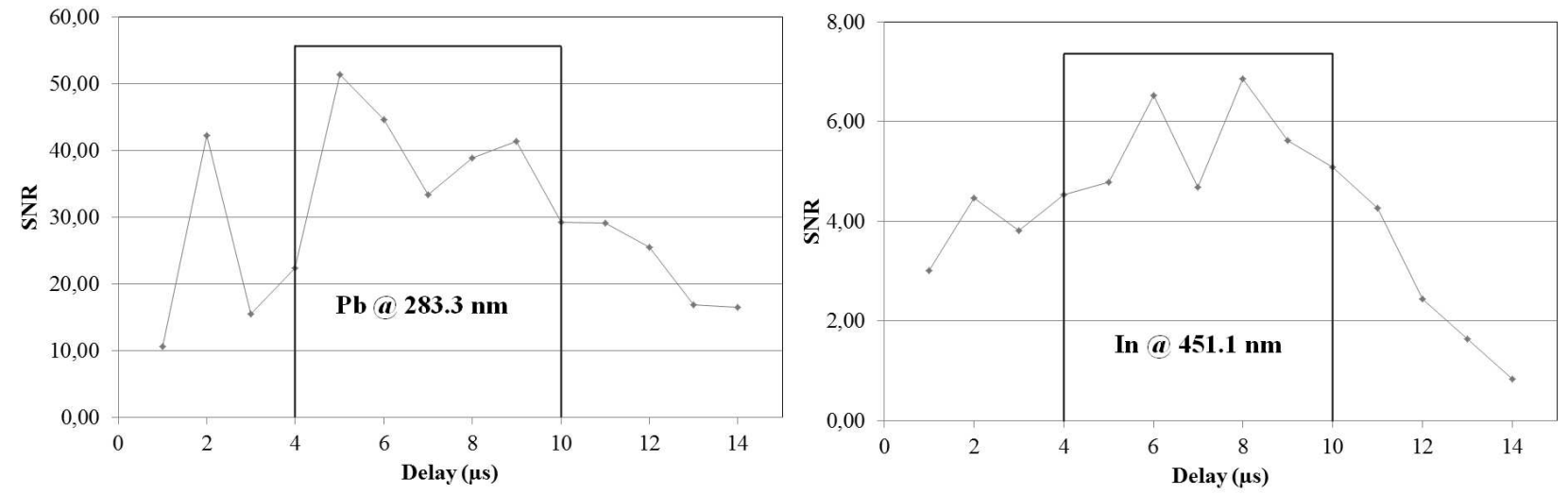

Figure 3: Signal to noise ratio for the $283.3 \mathrm{~nm}$ line of $\mathrm{Pb}([\mathrm{Pb}]=1365 \mathrm{ppm})$ and the $451.1 \mathrm{~nm}$ line of In $([\mathrm{In}]=50 \mathrm{ppm})$. Each point corresponds to the acquisition of spectra with a gate width of $1 \mu \mathrm{s}$. The optimal acquisition gate is shown in black.
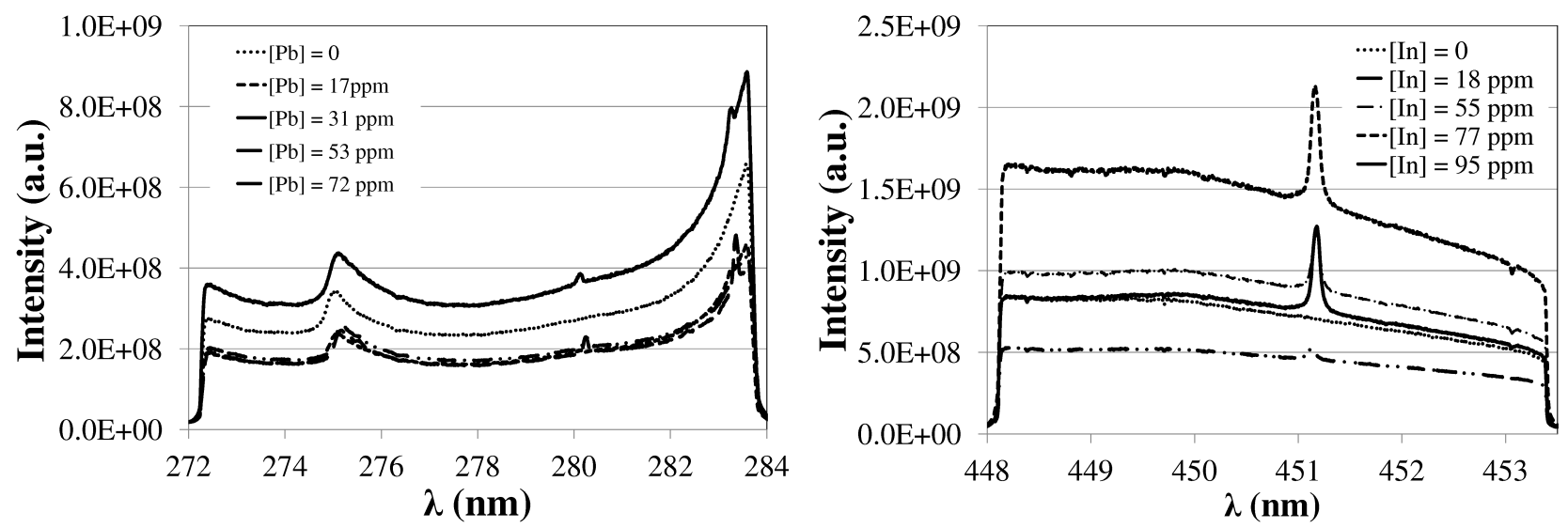

Figure 4: LIBS spectra of lead and indium in liquid sodium for the successive standard additions. Each spectrum is the result of 40000 laser shots accumulation. 

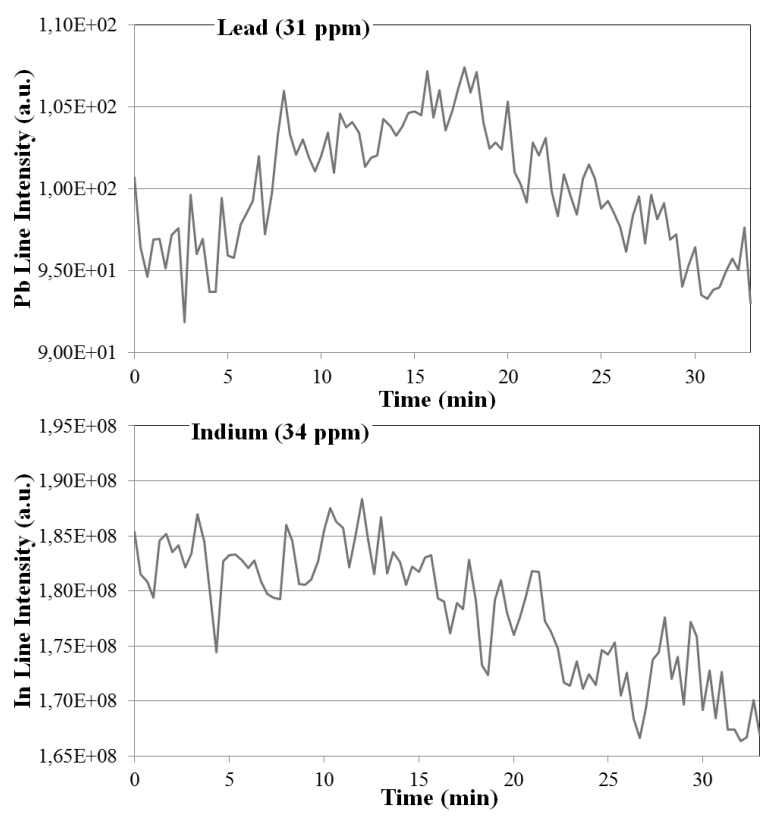

Figure 5: Examples of intensity drifts observed on the lead spectra (top figure) and indium spectra (bottom figure) during the acquisition of 100 spectra, each resulting from the accumulation of 400 laser shots.

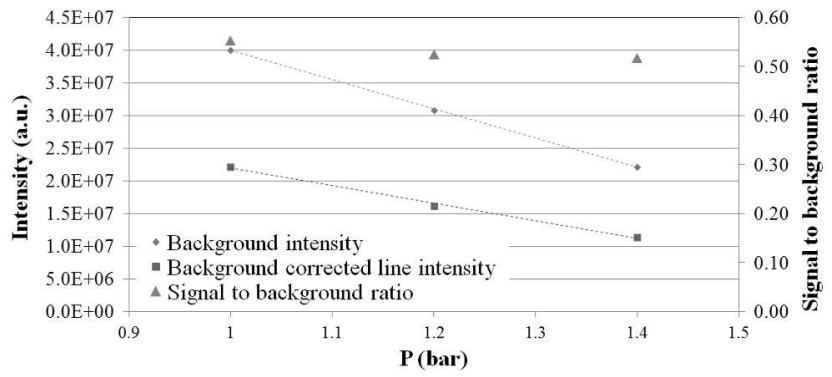

Figure 6: Evolution of the background-subtracted line intensity, of the background intensity, and of the signal to background ratio as a function of the pressure in the oven, for the indium $451.1 \mathrm{~nm}$ line. For every pressure value, the acquisition parameters used were those optimized in paragraph 2.2 . 
LEAD
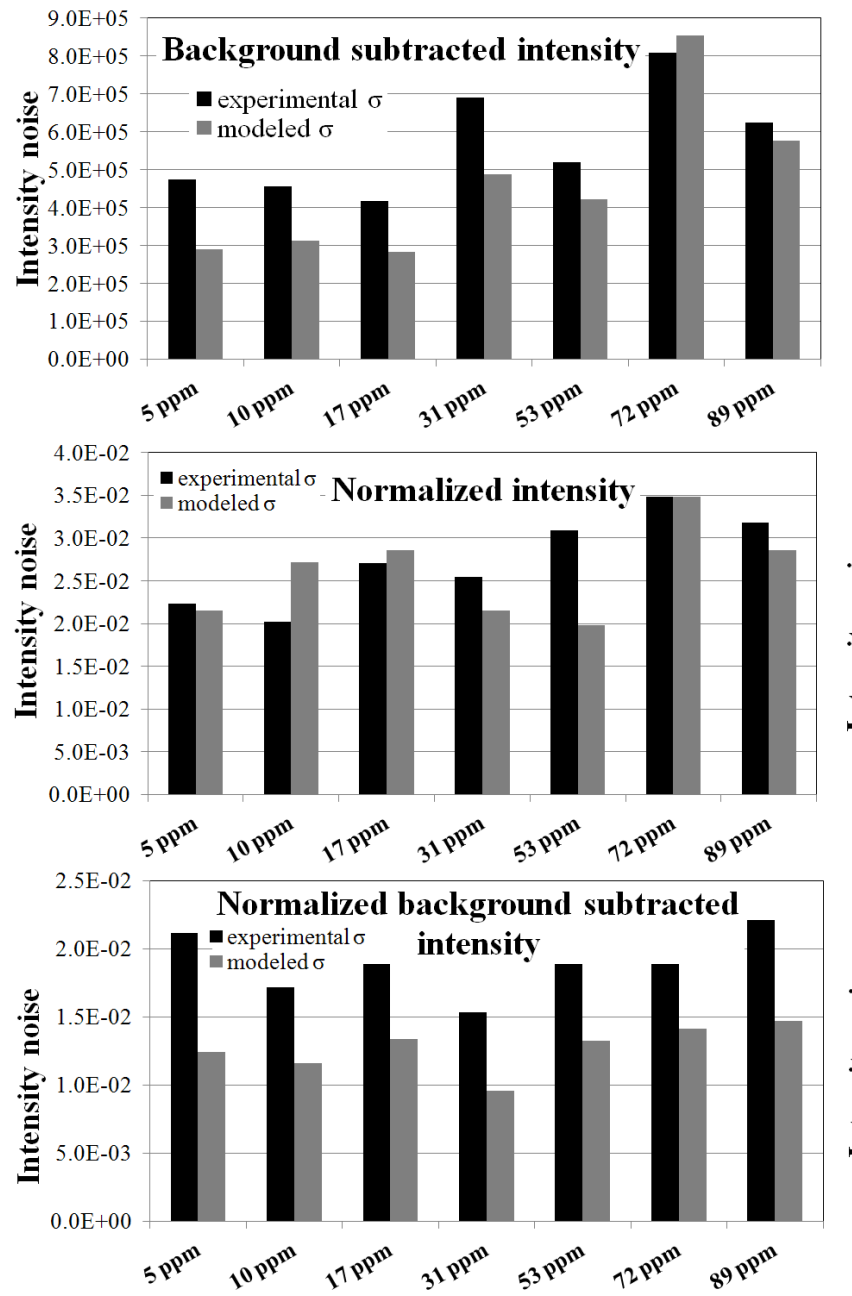

INDIUM
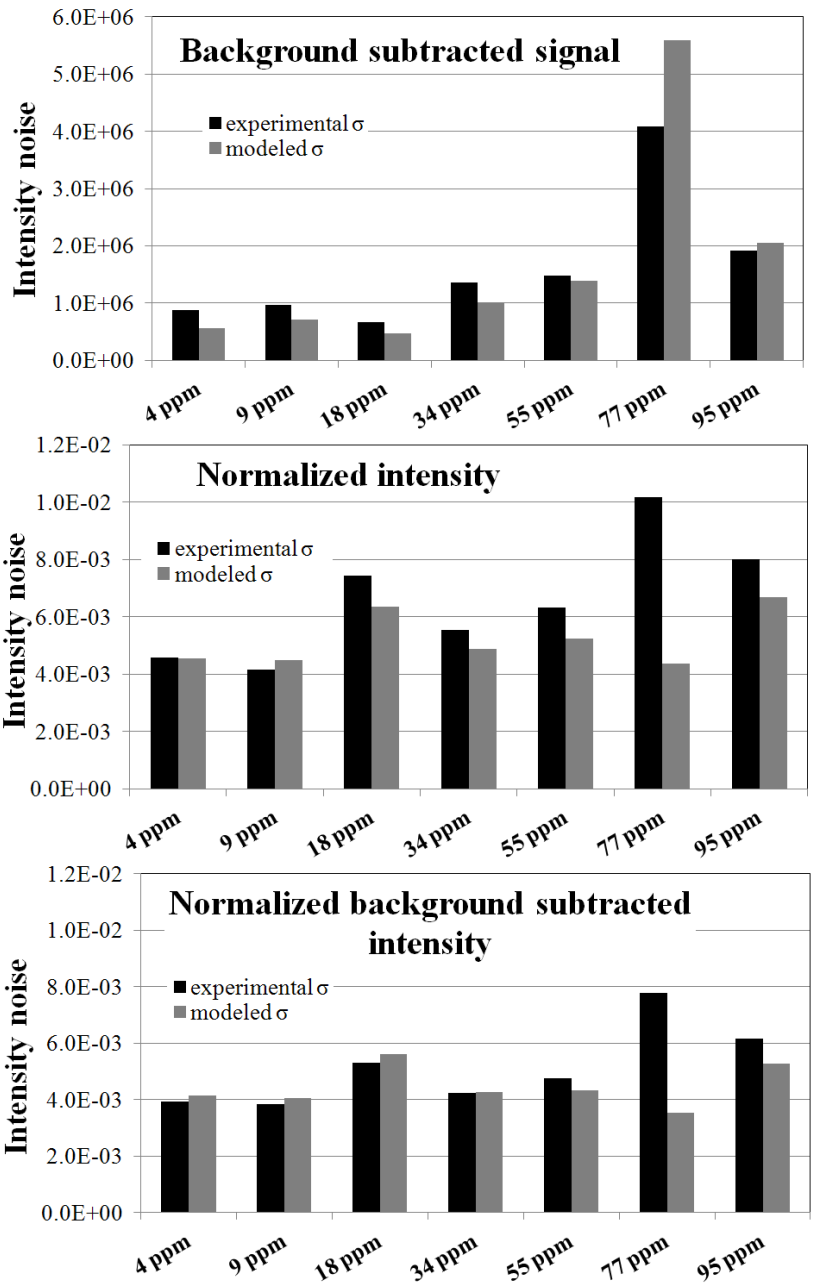

Figure 7: Comparison between experimental noises and noises calculated using the model applied to the spectra. The left hand side of the figure shows the results for lead, and the right hand side shows those for indium. 

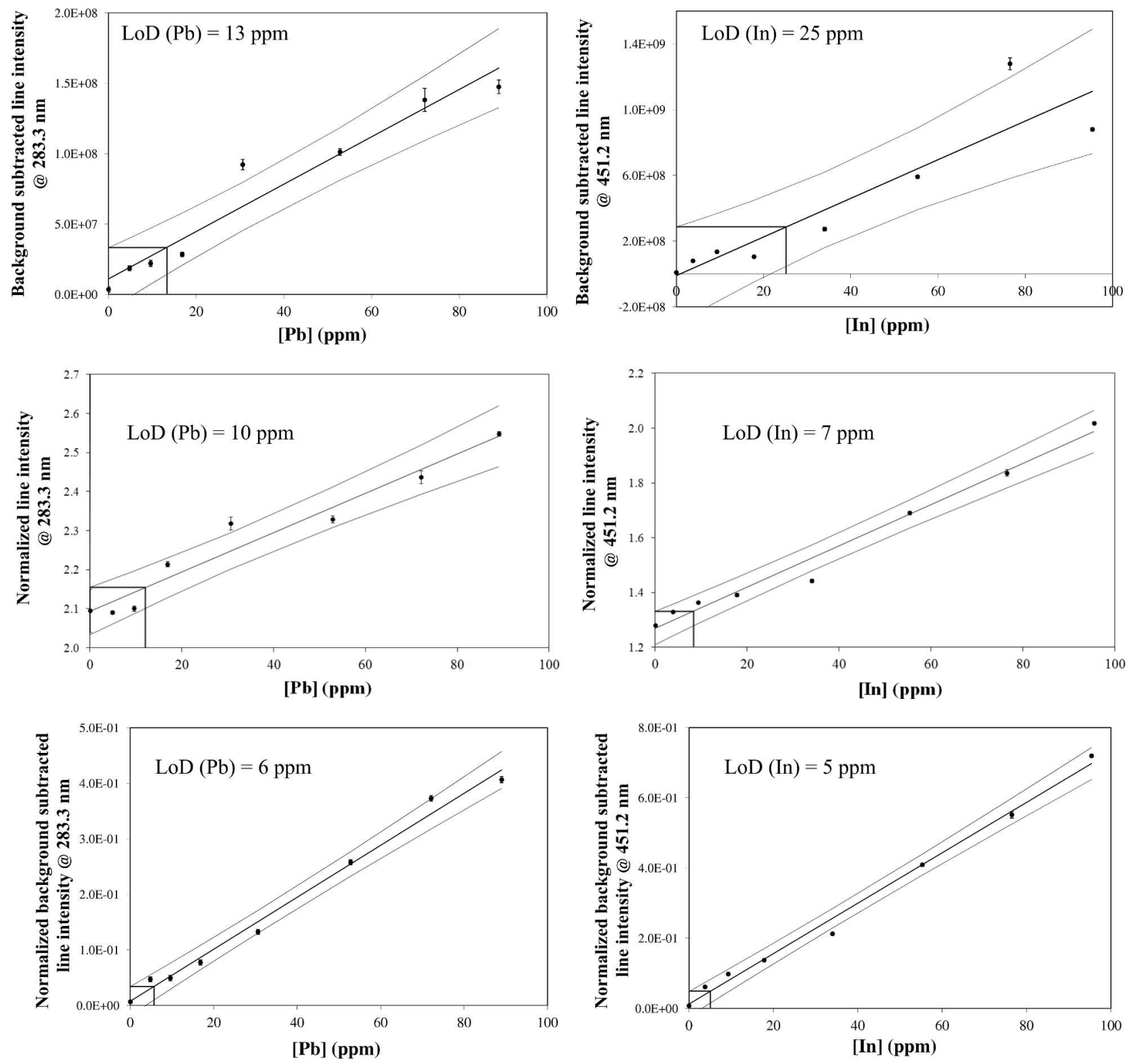

Figure 8: Calibration curves for the background-subtracted line intensity (top figures), the normalized raw intensity (middle figures) and normalized background-subtracted line intensity (bottom figures) of lead (left handside figures) and indium (right handside figures) in liquid sodium. Each point is the result of the accumulation of 4000 laser shots. The prediction bands are drawn for a $95 \%$ confidence level and are calculated from 10 replicas per concentration. 
${ }^{*}$ Highlights (for review)

- Direct analysis of liquid sodium purity is performed using LIBS

- Calibration lines are obtained using the standard addition method

- Detection limits are calculated for lead and indium

- Intensity drifts are observed due to pressure variations

- Drifts are modeled and corrected to decrease the analytical signal noise 